\title{
Brown Vialetto Van Laere syndrome: presenting with left ventricular non-compaction and mimicking mitochondrial disorders
}

\author{
Berna Şeker Yılmaz ${ }^{1,2}$, Serdar Ceylaner ${ }^{3 \oplus}$, Neslihan Önenli Mungan ${ }^{4 \oplus}$ \\ ${ }^{1}$ Genetics and Genomic Medicine Department, University College London, London, United Kingdom; ${ }^{2}$ Department of Pediatric \\ Metabolism, Mersin University Faculty of Medicine, Mersin; ${ }^{3}$ Department of Genetics, Intergen Genetics Centre, Ankara; ${ }^{4}$ Department \\ of Pediatric Metabolism, Çukurova University Faculty of Medicine, Adana, Turkey.
}

\begin{abstract}
Background. Brown-Vialetto-Van Laere syndrome (BVVLS) is a rare, treatable neurodegenerative disorder with a variable clinical presentation, caused by mutations in three different riboflavin transporter genes.

Case. An 11-year-old-boy presented with respiratory insufficiency and a rapidly progressive muscle weakness. He was the fifth child of a consanguineous marriage with a medical history of hearing loss. He was peripherally week with a reduced muscle tone. Upper extremity muscles were effected more than lower limbs. He deteriorated rapidly and became quadriplegic. Brain magnetic resonance imaging and magnetic resonance spectroscopy were normal. Echocardiography revealed left ventricular non-compaction. A homozygous c.1088C $>$ T (p.363L) missense mutation was identified in SLC52A2 gene. Significant clinical improvement was seen with high dose riboflavin.

Conclusion. This is the first reported BVVLS case presented with left ventricle-non compaction which may be caused by a secondary respiratory chain deficiency. Riboflavin transporter deficiencies should be considered in the differential diagnosis of mitochondrial disorders and secondary respiratory chain deficiencies should be thought during the follow-up of BVVLS.
\end{abstract}

Key words: Brown-Vialetto-Van Laere syndrome, riboflavin, left ventricle-non compaction, mitochondrial disorders.

Brown-Vialetto-Van Laere syndrome (BVVLS), a rare motor neuron disorder, is caused by three different riboflavin transporter (RFVT) genes; SLC52A3, SLC52A2, and SLC52A1 which codes RFVT3, RFVT2 and RFVT1, respectively. ${ }^{1-3}$ While SLC52A3 and SLC52A2 are the main responsible genes, there is only one neonatal case with a mutation in SLC52A1 gene, probably due to the defective placental expression. ${ }^{2}$ Defects of RFVTs, decreases cellular uptake of riboflavin and may cause defects in energy production and cellular function. ${ }^{2}$ BVVLS is in the same spectrum with Fazio-Londe syndrome, which

\footnotetext{
$\bowtie \quad$ Berna Şeker Yılmaz

b.yilmaz@ucl.ac.uk
}

Received 29th June 2020, revised 31st October 2020, accepted 10th November 2020. may present with cranial nerve palsies, bulbar palsy, respiratory compromise, sensorineural deafness, limb weakness and upper motor signs. ${ }^{1,4}$ There are some phenotypical differences between RFVT3 and RFVT2 deficiencies. While hearing loss and muscle weakness are the most common presenting symptoms, abnormal gait and/or ataxia is often a presenting feature of RFVT2 deficiency. ${ }^{5}$ Although the vast majority of patients are presented in early childhood, late onset presentation is more characteristic for a SLC52A3 mutation. ${ }^{5}$ Optic atrophy, retinitis pigmentosa, intellectual delay and nystagmus have been reported as other rare neurological features. ${ }^{6}$ Moreover, there are several other accompanying non-neurological findings such as diabetes insipidus, hypogonadism and hypertension. ${ }^{6}$ Due to this clinical variability, 
patients may initially be misdiagnosed. They are suspected to have other neurological diseases such as mitochondrial myopathies and they experience a longer clinical journey. ${ }^{7}$ Patients may also have abnormal acyl carnitine profiles, mimicking multiple acyl-CoA dehydrogenase deficiency. ${ }^{8}$ Some of the current publications report varying degrees of improvement in clinical outcome or stabilisation in the symptoms with high doses of riboflavin replacement especially when rapid treatment started in the earlier stages. ${ }^{1,9-11}$

Here we describe the clinical course and responsiveness to riboflavin with leftventricular non-compaction which is a rare manifestation of mitochondrial diseases and has never been reported in BVVLS.

\section{Case Report}

An 11-year-old-boy was admitted to our paediatric intensive care unit because of respiratory insufficiency and a rapidly progressive muscle weakness. He was the fifth child of a consanguineous marriage and there was a history of early infantile death in the elder two male siblings after a similar respiratory deficiency. His past medical history was unremarkable except some minor hearing difficulties in the previous 2 years. He had been seen by a general practitioner and hearing loss was thought to be associated with infections. Audiological examination was suggested but the family did not consider it and it has been dismissed. During the admission, he was in the status of respiratory failure requiring intubation and mechanical ventilation. He was peripherally weak with a reduced muscle tone. Upper extremity muscles were more involved than the lower extremities and he suffered from shoulder girdle and distal hand muscle weakness. Deep tendon reflexes were bilaterally absent at the knees and ankles. Babinski sign was bilateral negative. Audiologic evaluation demonstrated neurosensorial loss. Within the next few days, he rapidly deteriorated, dysphagia progressed and a feeding tube was also placed for nutrition. He became quadriplegic and could not walk. Ammonia, serum lactic acid, hepatorenal function, thyroid function tests and microelements were normal. His acyl carnitine profile and amino acid screens were also normal. His urine organic acid analysis showed mild etylmalonic and suberic aciduria. Cerebrospinal fluid protein levels were mildly elevated but it was acellular. The findings from electroencephalogram and ophthalmology assessments were normal. Brain magnetic resonance imaging (MRI) and magnetic resonance spectroscopy (MRS) were also non-significant. Echocardiography revealed left ventricular non-compaction without any cardiac anomalies. There were no changes in the electrocardiogram. He was initially diagnosed with a post infectious neuropathy and due to the possible autoimmune aetiology intravenous immunoglobulin and corticosteroid treatments were given. There was no significant improvement and based on the concern that his phenotype was compatible with a mitochondrial disorder, coenzyme Q10, riboflavin and carnitine were commenced. Despite this mitochondrial cocktail, there was no clinical improvement. Considering BVVLS in the differential diagnosis, oral riboflavin therapy was gradually increased in two weeks to $75 \mathrm{mg} / \mathrm{kg} /$ day. One week after increased riboflavin therapy the child was extubated to room air, two weeks later was feeding orally and in a month he was walking without any support, he has independent mobility. There are no reported side effects.

According to the clinical features and this dramatic response to riboflavin treatment, BVVLS was suspected. Peripheral venous blood was collected from the proband and his parents. A homozygous c.1088C $>$ T (p.P363L) missense mutation was identified in SLC52A2 gene (Fig. 1). Both parents were heterozygous for this mutation. This sequence change replaces proline with leucine at codon 363 of the protein (p.Pro363Leu). It has been classified as likely pathogenic by the ACMG guideline. 


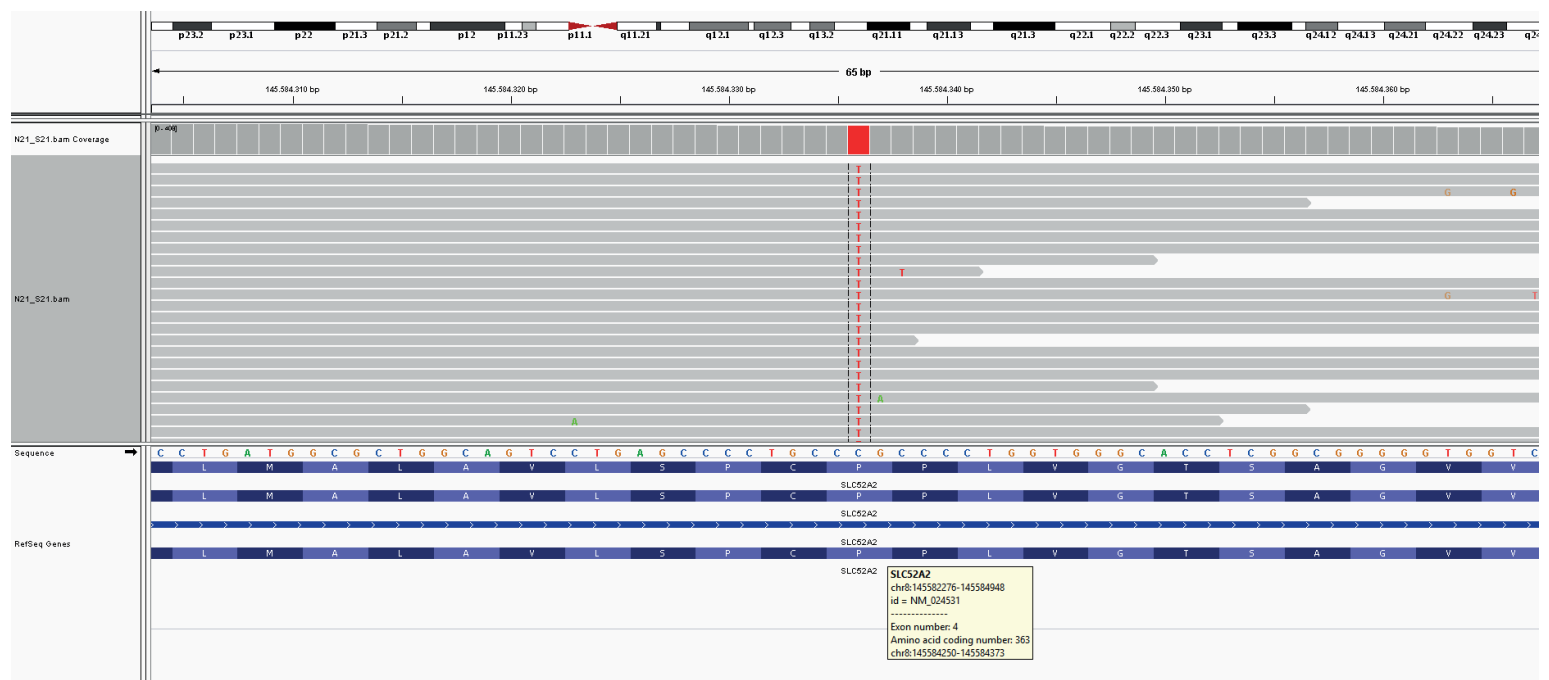

Fig. 1. c.1088C $>T$ (p.363L) mutation in the SLC52A2 gene.

Genetic analyses in this study were approved by the ethics committees of Cukurova University. Written informed consent was obtained from both of the patient's parents.

\section{Discussion}

BVVLS is a rare, neurodegenerative disorder characterised by progressive pontobulbar palsy, sensorineural deafness and respiratory insufficiency. ${ }^{11}$ There is a clinical heterogeneity; while milder forms present in the second or third decade, severe forms may die in the early infantile period..$^{3,6}$ Mean age of presentation is (4.1-8.2 years). ${ }^{1,2}$ Our patient presented slightly older than this when we consider the minor hearing difficulties as the main presenting symptom. Besides the variability in age of onset, clinical findings may differ from patient to patient including the characteristic features of lower cranial nerve involvement (nerves VIII-XII), lower and upper motor neurone limb signs, feeding difficulties and respiratory failure, facial weakness, sensory ataxia and optic atrophy. ${ }^{1,6}$ Here, in our case sensorineural hearing loss was an insidious feature, thus the clinical picture has been dismissed till the development of respiratory insufficiency. Therefore, the diagnosis and the treatment were delayed.
There are clinical differences in the phenotype of patients depending on the gene which has the causative mutation. Early onset and prominent weakness in the upper extremities is almost always seen in patients with mutations in SLC52A2, in contrast to those patients with SLC52A3 mutations, in whom the onset of weakness is often more generalized. ${ }^{3,8,9}$ Another typical presentation with $S L C 52 A 2$ mutations is the absence of upper motor neuron signs in the lower limbs which is very common for SLC52A3 mutations. ${ }^{4}$ Correlated with that, our patient had a more significant weakness in upper limbs.

Manole et al. ${ }^{12}$ reported a Brazilian girl who presented with sensorineural hearing loss, optic atrophy, respiratory insufficiency and sensorimotor neuropathy. She was compound heterozygous, besides c. $1088 \mathrm{C}>\mathrm{T}$ she had c.383C $>$ T mutation in the SLC52A2 gene. ${ }^{12} \mathrm{Her}$ phenotype was similar to our patient except the optical atrophy.

The flavoproteins which contain a nucleic acid derivative of riboflavin acts essentially in normal respiratory chain functions, and especially prominent for complex II- succinate dehydrogenase. ${ }^{13}$ Foley et al. ${ }^{9}$ reported two patients who had proven respiratory chain deficiency. One of them had slightly decreased complex IV activity (0.012; reference range: 
0.014-0.034) and the other one had decreased complex I activity (0.089; reference range: 0.104-0.268). ${ }^{9}$ They both had mutations in the SLC52A2 gene. Nimmo et al. $^{13}$ reported two patients who have complex II deficiency and red ragged fibres in histochemistry of muscle biopsy consistent with mitochondrial myopathy with both SLC52A2 and SLC52A3 mutations. Our patient had a phenotype which is suggestive for a mitochondrial myopathy. Although we could perform neither a muscle biopsy nor a respiratory chain enzyme activity measurement, this could be because of a possible respiratory chain enzyme deficiency. Thus, in the differential diagnosis of neuromyopathy, as well as the mitochondrial disorders riboflavin transporter defects should be considered in the early stages and high doses of riboflavin treatments should be given.

Cardiac presentation is not common for BVVLS. Several cardiac complications have been reported due to the hypoxia caused by respiratory insufficiency, diaphragmatic weakness or prolonged seizures. ${ }^{14}$ The cardiac finding of our patient, non-compaction of the left ventricle, has not been previously reported among patients with BVVLS. Left ventricular non-compaction is a rare manifestation of mitochondrial disorders mostly related with mitochondrial complex II deficiencies and Barth syndrome. ${ }^{15}$ This is the most interesting finding of our patient which may be due to a secondary mitochondrial defect.

In conclusion, this case highlights the importance of suspicion on the way to the early diagnosis. This led to early initiation of high dose riboflavin which probably provided a significant improvement in our case. Lifesaving treatment should be started immediately without awaiting the genetic confirmation. Further studies will clarify the underlying mitochondrial defects in the metabolic pathway. In addition, genetic counselling and genetic screening should be recommended to affective families. Prophylactic riboflavin treatment may be beneficial for preventing symptoms.

\section{REFERENCES}

1. Bosch AM, Stroek K, Abeling NG, Waterham HR, Ijlst L, Wanders RJ. The Brown-Vialetto-Van Laere and Fazio Londe syndrome revisited: natural history, genetics, treatment and future perspectives. Orphanet J Rare Dis 2012; 7: 83.

2. Jaeger B, Bosch AM. Clinical presentation and outcome of riboflavin transporter deficiency: mini review after five years of experience. J Inherit Metab Dis 2016; 39: 559-564.

3. Green P, Wiseman M, Crow YJ, et al. BrownVialetto-Van Laere syndrome, a ponto-bulbar palsy with deafness, is caused by mutations in c20orf54. Am J Hum Genet 2010; 86: 485-489.

4. Dipti S, Childs AM, Livingston JH, et al. BrownVialetto-Van Laere syndrome; variability in age at onset and disease progression highlighting the phenotypic overlap with Fazio-Londe disease. Brain Dev 2005; 27: 443-446.

5. O'Callaghan B, Bosch AM, Houlden H. An update on the genetics, clinical presentation, and pathomechanisms of human riboflavin transporter deficiency. J Inherit Metab Dis 2019; 42: 598-607.

6. Sathasivam S. Brown-Vialetto-Van Laere syndrome. Orphanet J Rare Dis 2008; 3: 9.

7. Amir F, Atzinger C, Massey K, et al. The clinical journey of patients with riboflavin transporter deficiency type 2. J Child Neurol 2020; 35: 283-290.

8. Bosch AM, Abeling NG, Ijlst L, et al. Brown-VialettoVan Laere and Fazio Londe syndrome is associated with a riboflavin transporter defect mimicking mild MADD: a new inborn error of metabolism with potential treatment. J Inherit Metab Dis 2011; 34: 159-164.

9. Foley AR, Menezes MP, Pandraud A, et al. Treatable childhood neuronopathy caused by mutations in riboflavin transporter RFVT2. Brain 2014; 137(Pt 1): 44-56.

10. Anand G, Hasan N, Jayapal S, et al. Early use of high-dose riboflavin in a case of Brown-Vialetto-Van Laere syndrome. Dev Med Child Neurol 2012; 54: 187-189.

11. Horoz OO, Mungan NO, Yildizdas D, et al. BrownVialetto-Van Laere syndrome: two siblings with a new mutation and dramatic therapeutic effect of high-dose riboflavin. J Pediatr Endocrinol Metab 2016; 29: 227-231.

12. Manole A, Jaunmuktane Z, Hargreaves I, et al. Clinical, pathological and functional characterization of riboflavin-responsive neuropathy. Brain 2017; 140: 2820-2837. 
13. Nimmo GAM, Ejaz R, Cordeiro D, Kannu P, Mercimek-Andrews S. Riboflavin transporter deficiency mimicking mitochondrial myopathy caused by complex II deficiency. Am J Med Genet A 2018; 176: 399-403.
14. da Silva-Junior FP, Moura Rde D, Rosemberg S Marchiori PE, Castro LH. Cor pulmonale in a patient with Brown-Vialetto-Van Laere syndrome: a case report. J Neurol Sci 2011; 300: 155-156.

15. El-Hattab AW, Scaglia F. Mitochondrial cardiomyopathies. Front Cardiovasc Med 2016; 3: 25. 